\title{
Amplitude Modulation Patterns of Local Field Potentials Reveal Asynchronous Neuronal Populations
}

\author{
Javier Díaz, ${ }^{1}$ Pablo Razeto-Barry, ${ }^{1}$ Juan-Carlos Letelier, ${ }^{1}$ John Caprio, ${ }^{3}$ and Juan Bacigalupo ${ }^{1,2}$ \\ ${ }^{1}$ Department of Biology, Faculty of Sciences, University of Chile, Santiago, Chile 7800023, and ${ }^{2}$ Institute of Cell Dynamics and Biotechnology, University of \\ Chile, Santiago, Chile 8370456, and ${ }^{3}$ Department of Biological Sciences, Louisiana State University, Baton Rouge, Louisiana 70803
}

\begin{abstract}
Neural oscillations, which appear in several areas of the nervous system and cover a wide frequency range, are a prominent issue in current neuroscience. Extracellularly recorded oscillations are generally thought to be a manifestation of a neural population with synchronized electrical activity resulting from coupling mechanisms. The vertebrate olfactory neuroepithelium exhibits $\beta$-band oscillations, termed peripheral waves (PWs), in their population response to odor stimulation. Here, we examine PWs in the channel catfish and propose that their properties could be explained as the superposition of asynchronous oscillators. Our model shows that the intriguing random pattern of amplitude-modulated PWs could be explained by Rayleigh fading, an interference phenomenon well known in physics and recognizable using statistical methods and signal analysis. We are proposing a mathematical fingerprint to characterize neural signals generated by the addition of random phase oscillators. Our interpretation of PWs as arising from asynchronous oscillators could be generalized to other neuronal populations, because it suggests that neural oscillations, detected in local field potential recordings within a narrow frequency band, do not necessarily originate from synchronization events.
\end{abstract}

Key words: peripheral waves; Rayleigh fading; olfactory epithelium; olfactory neuron; Kuramoto model; neuronal synchronization

\section{Introduction}

For over 15 years, neuronal synchronization has been considered a fundamental step in sensory, motor, and cognitive processing (Buzsaki and Draguhn, 2004; Lledo et al., 2005). In some systems, neuronal synchronization was detected by explicit crosscorrelation between spike trains, but in many studies, it was inferred by the existence of narrowband oscillations in recorded local field potentials (LFPs) (Engel et al., 2001; Varela et al., 2001). The rationale for this identification is the assumption that narrowband oscillations must necessarily reflect the constructive summation of synchronous oscillators. Thus, a great amount of theoretical effort was directed to elucidate the coupling mechanism(s) producing synchronous firing (White et al., 1998; Ermentrout and Chow, 2002; Jones, 2002; Averbeck and Lee, 2004); however, this search can be expanded by taking into account aspects such as a statistical analysis of the degree of synchrony of the underlying oscillator population or an estimation of the number of oscillators involved. For example, very different phenomena can arise in LFP recordings, which involve only a mesoscopic quantity of oscillators (few tens of oscillators) when compared

Received April 13, 2006; revised June 5, 2007; accepted June 6, 2007.

This work was supported by Comisión Nacional de Investigación Cientifica y Tecnológica Programa Bicentenario en Ciencia y Tecnología ACT 45, Ministerio de Planificación Iniciativa Científica Milenio P05-001-F (J.B.), Fondo Nacional de Desarrollo Científico y Tecnológico 1061108 (J.-C.L.), and Grant National Science Foundation IBN0314970 (J.C.).J.B. holds a John S. Guggenheim Memorial Foundation Fellowship. We are indebted to Ranulfo Romo and Francisco Bezanilla for critical reading of this manuscript, Magdalena Sanhueza for invaluable discussions, Oliver Schmachtenberg for advice, and the important input of the anonymous reviewers.

Correspondence should be addressed to Juan Bacigalupo, Department of Biology, University of Chile, Las Palmeras 3425, Nunoa, Santiago, Chile 7800023. E-mail: bacigalu@uchile.cl.

DOl:10.1523/JNEUROSCI.4512-06.2007

Copyright $\odot 2007$ Society for Neuroscience $\quad$ 0270-6474/07/279238-08\$15.00/0 with macroscopic techniques (EEG), where millions of oscillators are involved. Furthermore, powerful and stereotypical neural oscillations can be detected in systems, like the vertebrate olfactory epithelium (OE), where no coupling mechanisms (interneurons, gap junctions) likely exist between the elementary oscillators. It is thus worthwhile to consider whether neural oscillations can arise in some circumstances by a mechanism not involving coupling. To test the hypothesis that neural oscillations can be generated by the summation of uncoupled or uncorrelated oscillators, we studied the statistical properties of "peripheral waves" (PWs), a classic case of neural oscillations found in the olfactory epithelium of the vertebrate (Ottoson, 1956; Nikonov et al., 2002).

Since the mid 1950s, it was known that potent odors induce strong, mainly $\beta$-band oscillations termed peripheral waves (Adrian, 1955; Ottoson, 1956) in local field potentials of the olfactory epithelium. Initially, PWs were described as "unstable signals" or "random shapes" (Takagi and Shibuya, 1961), because they exhibit amplitude modulation patterns that never repeat themselves (see Fig. 1A). The current view is that PWs originate from neural activity within the olfactory organ and not by centrifugal influence or electrotonic spread from the olfactory bulb, because transection of the olfactory nerve does not eliminate the waves (Sutterlin and Sutterlin, 1971; Dorries and Kauer, 2000; Nakazawa et al., 2000). Although examples of PW are abundant (Nikonov et al., 2002), their generating mechanisms, as well as their possible function(s), are still unclear. The dominant theoretical notion is that PWs represent intermittent synchronous activity of groups of olfactory receptor neurons (ORNs) (Ottoson, 1956); however, because there are no established neural connections between ORNs, the mechanism for this purported peripheral synchrony remains unclear, and PWs have been linked to gaseous 

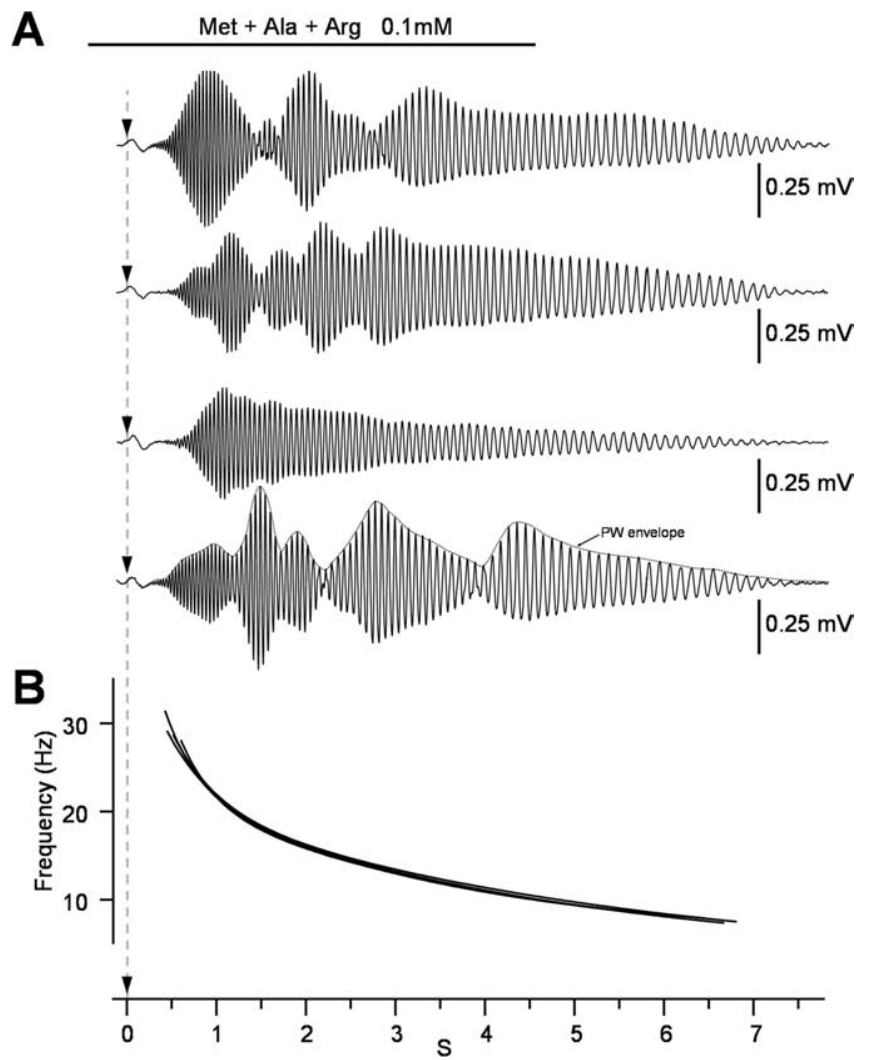

Figure 1. Fundamental properties of PWs. A, Examples of four PWs recorded from the olfactory epithelium of channel catfish (Nikonov et al., 2002) (Ictalurus punctatus) during odorant stimulation [5 s pulse of a mixture of the L-isomers of 0.1 mm methionine (met), arginine (arg), and alanine (ala)]. PWs are shown aligned to the start of the EOG (arrowheads), which were removed by high-pass filtering (Nikonov et al., 2002). The bottom trace shows a PW with its envelope calculated by Hilbert transform. $\boldsymbol{B}$, Instantaneous frequencies (calculated by a Hilbert transform method and fitted by a double exponential) for the PWs shown in $\boldsymbol{A}$ varied almost identically. The time scale applies to $\boldsymbol{A}$ and $\boldsymbol{B}$.

second messengers, gap junctions, ephaptic interactions, and electric field effects (Tucker, 1975a,b; Dorries and Kauer, 2000; Suzuki et al., 2004). PWs provide an appropriate framework to test our model, which considers the possibility that neural oscillations may arise from the random addition of individual uncoupled oscillators.

\section{Materials and Methods}

Experimental procedures. Channel catfish (Ictalurus punctatus) were anesthetized and manipulated according to the guidelines of the Animal Ethics Committee of Louisiana State University. Extracellular recordings and stimulation of the catfish olfactory epithelium were performed as described previously (Parker et al., 2000; Nikonov et al., 2002). The stimulus used in this study was a mixture of the L-isomers of $0.1 \mathrm{~mm}$ methionine, arginine, and alanine applied for $5 \mathrm{~s}$. A minimum of 3 min interstimulus intervals (ISIs) were used to avoid adaptation. Low impedance $(\sim 50 \mathrm{~K} \Omega$ ) Wood's metal microelectrodes plated with platinum were used. LFPs were amplified, bandpass filtered between 3 and $300 \mathrm{~Hz}$, and stored on magnetic tape. After acquisition (sampled at $10 \mathrm{kHz}$ ), the signal was digitally filtered between 3 and $80 \mathrm{~Hz}$.

Eight sequential presentations of identical stimuli to the same animal produced eight instances of PWs, and their slow-varying envelopes, $A(t)$, were calculated using the Hilbert transform. For each set of eight envelopes $A(t)$, the average across repetitions $[\bar{A}(t)]$, and the SD (also across repetitions) $\sigma_{A}(t)$ were calculated. The coefficient of variation $(\mathrm{CV})$ over time $\mathrm{CV}(t)$ [defined as $\left.\sigma_{A}(t) / \bar{A}(t)\right]$ was calculated, as well as the time average $\langle\mathrm{CV}(t)\rangle$ (Fig. $1 A$, bottom trace).

Kuramoto and Matthews-Mirollo-Strogatz model systems. Using
Kuramoto dynamics (Strogatz, 2000; Izhikevich and Kuramoto, 2006), we simulated artificial LFPs generated by the superposition of signals from a population of 25 weakly coupled oscillators. To obtain variable degrees of coupling, we set the Kuramoto's coupling strength $K$ to different values: 0 (corresponding to no coupling), 2, 4, 6, ., 42 (corresponding to a very tight coupling). For each simulated set of LFPs (eight elements), we calculated the same statistical values described for real signals. Simulated LFP signals using the Matthews-Mirollo-Strogatz model (MMS) regimes were also calculated for $n=25$ oscillators with coupling parameter between $K=0$ (random) and $K=1.7$ (high degree of coupling) in steps of 0.005 . The bandwidth $(\Delta)$ varied between 0.2 and $1.8 \mathrm{~Hz}$ in $0.025 \mathrm{~Hz}$ steps. Differential equations, described by Matthews et al. (1991), were numerically solved using fourth-order Runge-Kutta methods coded in Igor (WaveMetrics, Lake Oswego, OR).

Summation of oscillators with variable frequency and amplitude. Artificial PWs were also obtained by the addition of uncoupled oscillators mimicking the amplitude and frequency signatures of empirical PWs (Fig. $1 B$ ). The amplitude profile of each oscillator changed as depicted in Figure $5 A$ (top trace), whereas their individual frequency was represented by realizations of the random process defined by the following:

$$
f(t)=y_{0}+A_{1} e^{-\tau_{1} t}+A_{2} e^{-\tau_{2} t},
$$

with the empirical values $y_{0}=2.94 \pm 0.30 ; A_{1}=6.27 \pm 3.00 ; A_{2}=$ $17.60 \pm 0.30 ; \tau_{1}=0.52 \pm 0.02$; and $\tau_{2}=4.760 \pm 0.001$ and a phase uniformly distributed in $[-\pi, \pi]$. This random process simulated the widespread frequency change during real PWs (Fig. $1 B$ ). Each artificial PW was generated by the addition of 25 oscillators.

Summation of spike trains. PWs were also simulated by the addition of uncorrelated spike trains. Each spike train $(m)$ was simulated by three random variables $\left(T_{m}, t_{0, m}\right.$, and a jitter $\left.{ }_{m}\right) . T_{m}$ corresponds to the average train ISI obtained from a Gaussian probability density function (PDF) $(33.3 \pm 0.6 \mathrm{~ms}) . t_{0, m}$ [simulated by a uniform random variable in the range $\left.\left(0, T_{m}\right)\right]$ corresponds to the time of the initial spike and thus serves to define an initial relative phase between trains. Neuronal noise was represented by jitter ${ }_{i}$, a zero mean Gaussian random variable. The timing $t_{i, m}$ for spike $i$ was recursively computed as follows:

$$
t_{i, m}=t_{i-1, m}+T_{m}+\text { jitter }_{i} .
$$

Finally, at $t_{i, m}$, a smoothed, real recorded spike template was inserted. These simulated trains served to illustrate the basic idea that PW can be generated by regular spiking activity. Each artificial PW was generated by the addition of 100 of such spike trains.

Statistical analysis. To obtain valid statistics to compare CV obtained from real signals from those generated by Kuramoto dynamics, we applied resampling methods. For each coupling strengths $K(0,2,4,6, \ldots$ 42), 13 groups of eight PWs were calculated. For each group of eight, we calculated their CV. To obtain their empirical probability density distribution, we resampled each population of $13 \mathrm{CV}$. This PDF was used to calculate confidence intervals (95 and 99\%) for the CV mean. We also resampled the set $(n=13)$ of CV experimentally obtained.

\section{Results}

PWs, which are triggered by intense odor stimuli, are $\beta$-band amplitude modulated (AM) signals (Fig. $1 A$ ), where their carrier frequency varies with a temporal profile that depends on stimulus properties (analysis not shown in this work). For the particular biologically relevant olfactory stimuli (i.e., amino acids) for fish used in this work, the instantaneous frequency of PWs (determined using the Hilbert transform) declined from 30 to $10 \mathrm{~Hz}$ and was well described as a double exponential function (Fig. $1 B)$. Although the pattern of amplitude modulation differs for each stimulus presentation (Fig. $1 \mathrm{~A}$ ), the frequency time course is strongly conserved (Fig. $1 \mathrm{~B}$ ).

Because no previous investigation was able to show electrical coupling between ORNs or demonstrate synchrony in responses from a population of ORNs, we developed an alternative hypoth- 
$30 \pm 0.1 \mathrm{~Hz}$

A

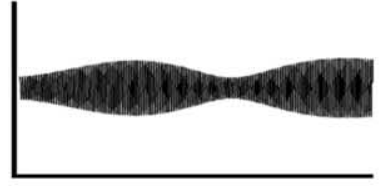

B

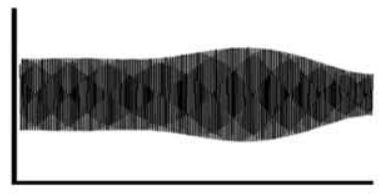

C

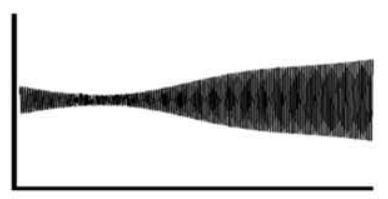

D
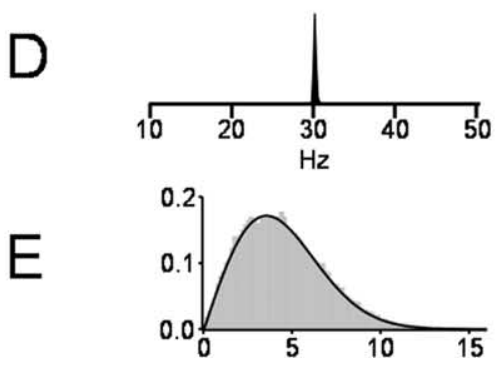

$30 \pm 0.5 \mathrm{~Hz}$
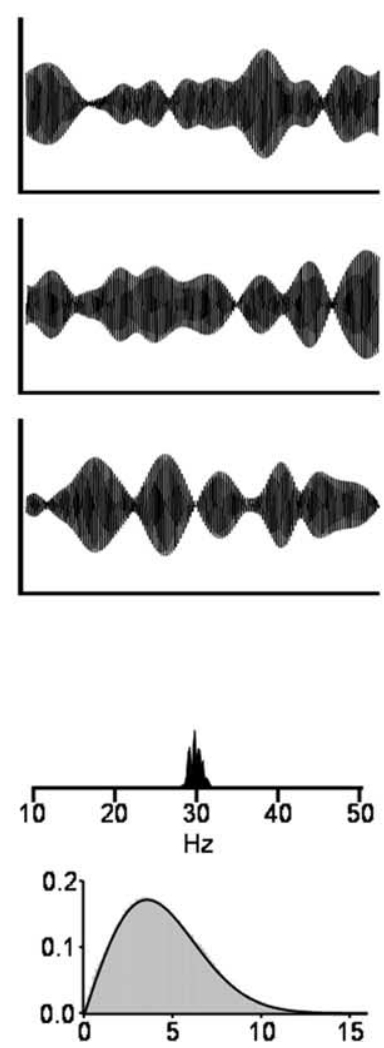

$30 \pm 1.5 \mathrm{~Hz}$
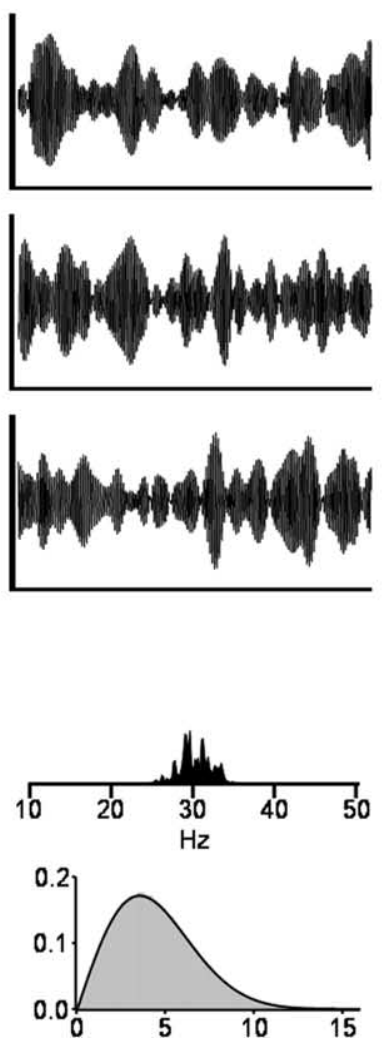

$30 \pm 5.0 \mathrm{~Hz}$
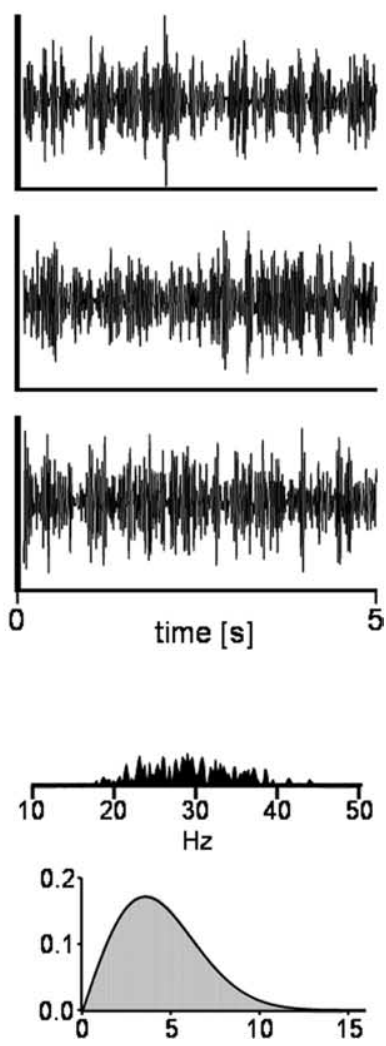

Figure 2. Rayleigh fading exhibits random amplitude modulation similar to PWs. The sum $\left[S_{N}(t)\right]$ of 25 sinusoidal oscillators of unitary amplitude with different bandwidths and random initial phases $\phi_{k}$ is shown. The frequency of each oscillator was chosen randomly from a Gaussian PDF centered at $30 \mathrm{~Hz}$ with SDs $\left(\sigma_{f}\right)$ of $0.1,0.5,1.5$, and $5 \mathrm{~Hz}$ as indicated at the top of each column. To illustrate the random nature of envelopes, $\boldsymbol{A}, \boldsymbol{B}$, and $\boldsymbol{C}$ display three different instances of $S_{N}(t)$. Power spectral density of $S_{N}(t)(\boldsymbol{D})$ and histogram $(\boldsymbol{E})$ of its envelope (using a $3600 \mathrm{~s}$ data segment) with the Rayleigh distribution overlapped is shown (black trace).

esis to explain the origin of PWs. The basis of our arguments is the fact, well known in statistical physics (Goodman, 1985), that the addition of $N$ sinusoidal oscillators as follows:

$$
S_{N}(t)=\sum_{k=1}^{N} a \sin \left(2 \pi f_{k} t+\phi_{k}\right),
$$

with amplitude $a$, and where the frequency $f_{k}$ of individual oscillators is obtained from a narrow frequency distribution centered in $f_{0}$ and dispersion $\sigma_{f}$ as follows:

$$
f_{k}=f_{0} \pm \sigma_{f}
$$

and the initial phases $\phi_{k}$ are uniformly distributed in $[-\pi, \pi]$, results in an AM-like signal as follows:

$$
S_{N}(t)=A(t) \sin \left[2 \pi f_{0} t+\beta(t)\right] .
$$

The signal $S_{N}(t)$ can be interpreted as a carrier, represented by $\sin \left[2 \pi f_{0} t+\beta(t)\right]$, multiplied by an envelope (or modulating) function $A(t)$, which may vary between 0 and $a \cdot N$. In addition, $A(t)$ follows a Rayleigh distribution with an RMS amplitude equal to $a \sqrt{N}$ (Papoulis, 1984). Furthermore, $A(t)$ has a spectrum depending on $\sigma_{f}$ and a characteristic temporal profile known as Rayleigh fading (Fig. 2), well known in the telecommunication industry and in statistical optics. In mobile communications, the multipath problem is an important technological challenge, because the multiple reflections of an electromagnetic wave reaching a receiver have random phases and slightly different frequen- cies, thus producing a time-varying pattern of constructive and destructive interferences displaying Rayleigh fading (Sklar, 1997). We thus propose that PWs result from the superposition of electric fields produced by a mesoscopic ensemble (Buzsaki, 2004) ( 15-100) of ORNs that fire in a constrained frequency band with random phases with respect to each other.

Although Rayleigh fading is the fingerprint of totally asynchronous oscillators, a more general statistical framework to describe intermediate situations where oscillators could be partially synchronized (Izhikevich and Kuramoto, 2006) is required to analyze LFP signals.

Adopting the phasor notation, each elementary oscillator is represented by a rotating vector $\vec{v}_{k}(t)$ having a magnitude $a$ and a time varying phase $\phi_{k}(t)$ as follows:

$$
\vec{v}_{k}(t)=a e^{i \phi_{k}(t)},
$$

then $A(t)$ [the envelope of wave $S_{N}(t)$ ] corresponds to the time varying magnitude of the vectorial summation of such phasors as follows:

$$
A(t)=\left|\sum_{k=1}^{N} \vec{v}_{k}(t)\right|
$$

and the envelope of an LPF is the magnitude of the vectorial sum of $\vec{v}_{k}(t)$. Although the relative phase difference among vectors $\vec{v}_{k}(t)$ changes moment by moment as the vectors rotate with different frequencies, an instantaneous phase dispersion $\hat{\sigma}_{\phi}(t)$ 

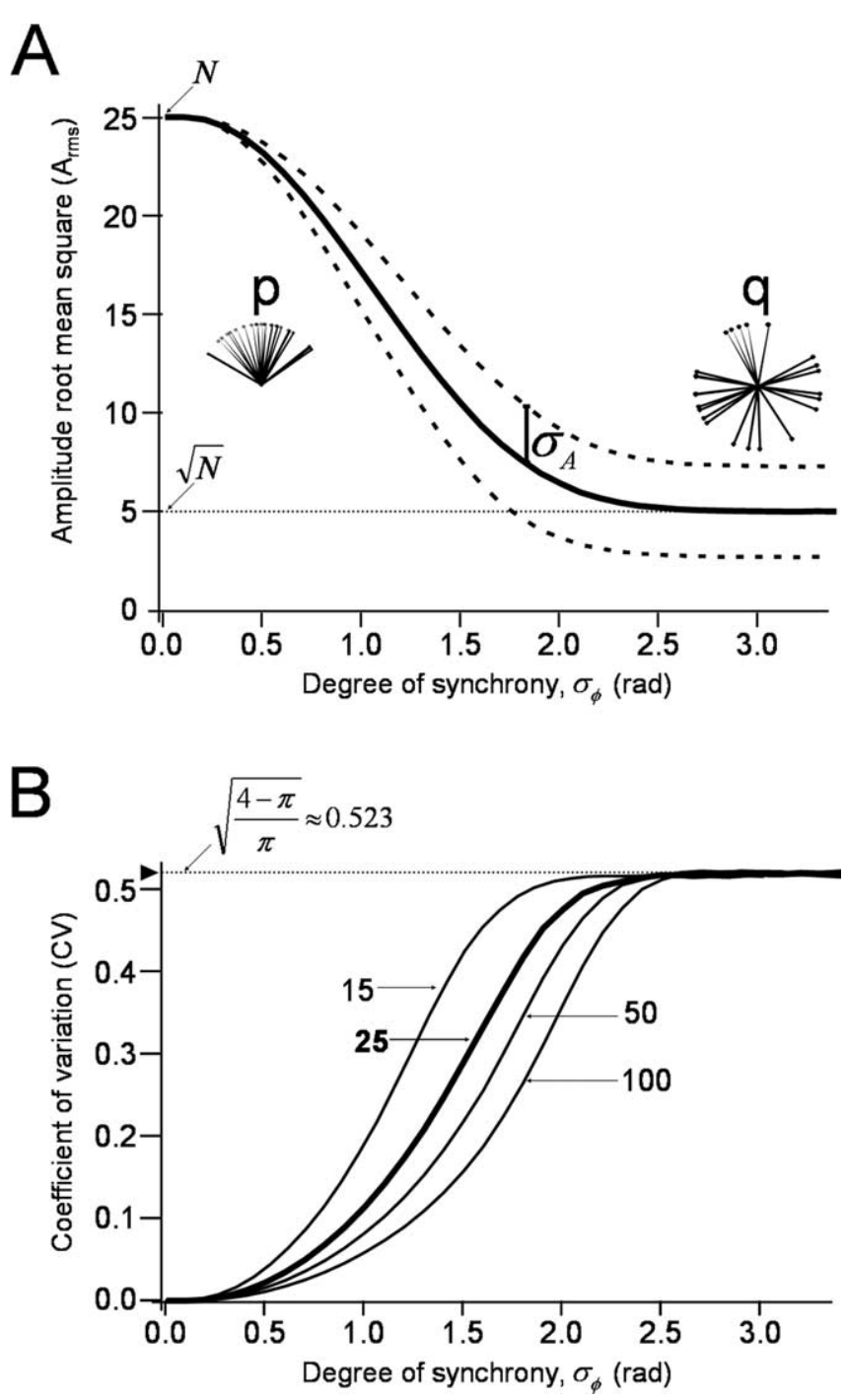

Figure 3. Statistical properties of the sum of oscillators with different degrees of synchrony. The vectorial sum of 25 unitary two-dimensional random vectors was calculated with phases taken from a zero mean Gaussian PDF with an SD of $\sigma_{\phi}$. For each $\sigma_{\phi}$ (x-axis), the rms amplitude $\left(A_{\mathrm{rms}}\right), \mathrm{SD}\left(\sigma_{A}\right)$, and $\mathrm{CV}$ of the resultant vector were calculated for a universe of $10^{5}$ trials. $A$, Relationship between the amplitude $\left(A_{\text {rms }}\right)$ and its dispersion $\left(\sigma_{A}\right)$ with respect to the degree of synchrony $\sigma_{\phi}$ (see Results) of the population of vectors. $p$ and $q$ represent instances of vectors orientations for $\sigma_{\phi}=0.5$ and 3.0 rad, respectively. $\boldsymbol{B}$, Relationship between $\mathrm{CV}$ and $\sigma_{\phi}$. The $\mathrm{CV}$ reaches an asymptotic value of 0.523 (arrowhead) when the unitary vectors are totally random. Thin lines represent alternative numbers of oscillators $(15,50$, or 100$)$.

can be defined from a snapshot of the orientations of the vectors (Fig. 3, p, q). The standard deviation, $\sigma_{\phi}$, of the probability density function (PDF) of the vectors' phases over time, defined as the time average $\sigma_{\phi}=\left\langle\hat{\sigma}_{\phi}(t)\right\rangle$, measures the degree of synchrony of the population of oscillators. Thus, the problem of quantifying the properties of a signal envelope, a time varying function, can be reduced to consider vectorial sums with different $\sigma_{\phi}$, thus subsuming time in a pure statistical framework.

Figure $3 A$ shows the rms amplitude $\left(A_{\mathrm{rms}}\right)$ and its $\operatorname{SD}\left(\sigma_{A}\right)$ versus phase dispersion $\left(\sigma_{\phi}\right)$ for the result of numerically modeled sums of $N=25$ oscillators. $\sigma_{\phi}$ changes from total synchrony $\left(\sigma_{\phi}=0\right)$, with $A_{\text {rms }}=N$, to total asynchrony $\left(\sigma_{\phi} \rightarrow \pi\right)$, a situation analogous to the two-dimensional random walk, yielding an $A_{\text {rms }}=\sqrt{N}$. For mesoscopic LFP recordings (i.e., small number of oscillators), $A_{\text {rms }}$ varies within the same order of magnitude than $N$, but as $N$ increases, the ratio $\sqrt{N} / N$ becomes negligible.
Figure $3 B$ shows the coefficient of variation of the envelope (CV $=\sigma_{A} / \bar{A} ; \bar{A}$ is the average envelope amplitude) for groups of $N=15,25,50$, and 100 oscillators. For intermediate values of $\sigma_{\phi}$, the profile of the curve depends on $N$, but for extreme values, it is independent of $N$. Because the $\mathrm{CV}$ of the Rayleigh distribution is $\sqrt{(4-\pi) / \pi} \approx 0.523$, as $\sigma_{\phi} \rightarrow \pi$, the CV approximates asymptotically the value of 0.523 , which can be interpreted as a fingerprint for the superposition of an asynchronous population of oscillators. Thus, the CV can be interpreted as a parameter measuring the degree of synchrony, because it covaries sigmoidally with $\sigma_{\phi}$ presenting two characteristic values: close to zero (synchronous oscillators) and close to 0.523 (asynchronous oscillators).

To infer the degree of synchrony in the network of oscillators that produce an AM-like signal, the $\mathrm{CV}$ of its envelope must be computed, ideally, over an infinitely long period. In experimental situations where signals are finite and the temporal profile of an individual oscillator is unknown, the empirical $\mathrm{CV}$ can be calculated from the experimental data as the time average of the ratio between the instantaneous SD and the instantaneous mean of a family of time-locked envelopes, obtained as repetitions of the same experiment (i.e., $\mathrm{CV}=$ $\left.\langle\mathrm{CV}(t)\rangle=\left\langle\sigma_{A i}(t) / \bar{A}_{i}(t)\right\rangle\right)$ (Fig. 4).

To test the hypothesis that PWs are an example of Rayleigh fading produced by uncoupled oscillators, we calculated the empirical $C V(t)$ for families of PW envelopes. Figure $4 A$ shows how the empirical $\mathrm{CV}(t)$ varied after stimulus onset for a family of eight PW traces. For these cases, $\mathrm{CV}(t)$ hovers around the theoretical value 0.523 , and its experimental time average is $\mathrm{CV}=$ 0.503 . Next, we calculated the CV for theoretical situations where oscillators were coupled to each other. To generate such surrogate data, we used the most common model in the literature, the Kuramoto model (Strogatz, 2000), for the synchronization of coupled oscillators. In this model, the frequency of each oscillator $\left(\phi_{i}\right)$ in the network depends on its natural frequency $\left(\omega_{i}\right)$ plus a perturbation produced by the collective influence of the rest of the network, controlled by a coupling strength parameter $K$ (Strogatz, 2000) as follows:

$$
\frac{d \phi_{i}}{d t}=\omega_{i}+K r \sin \left(\psi-\phi_{i}\right),
$$

where $r$ and $\psi$ correspond, respectively, to the magnitude and phase of the normalized vectorial summation of the phasors representing individual oscillators. The polar coordinates of this mean field vector (also known as order parameter or centroid) can be obtained by the following:

$$
\begin{gathered}
r=\sqrt{\left(\sum_{i=1}^{N} \cos \left(\phi_{i}\right)\right)^{2}+\left(\sum_{i=1}^{N} \sin \left(\phi_{i}\right)\right)^{2}}, \\
\psi=\arctan \left(\frac{\sum_{i=1}^{N} \sin \left(\phi_{i}\right)}{\sum_{i=1}^{N} \cos \left(\phi_{i}\right)}\right)
\end{gathered}
$$

Thus, we simulated the resulting PWs by adding 25 oscillators under different Kuramoto coupling strengths and calculated their CV (Fig. 4B). The Kuramoto model features a bifurcation 


\section{A}

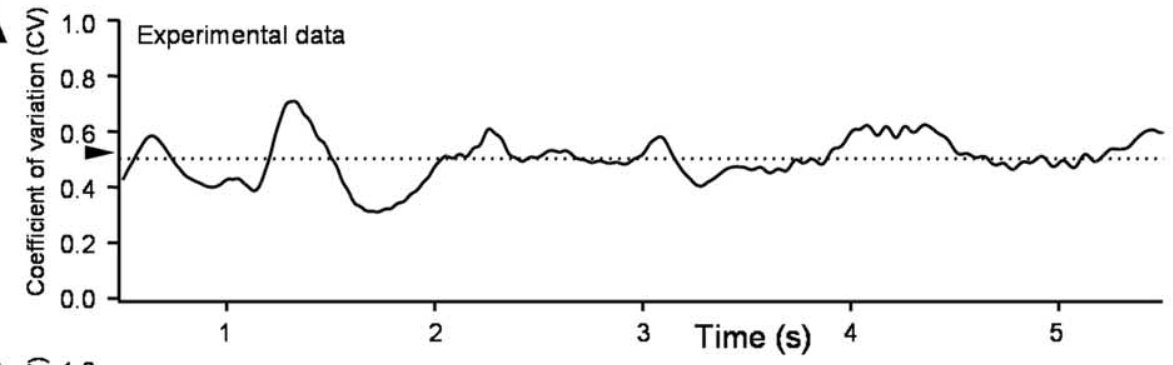

B
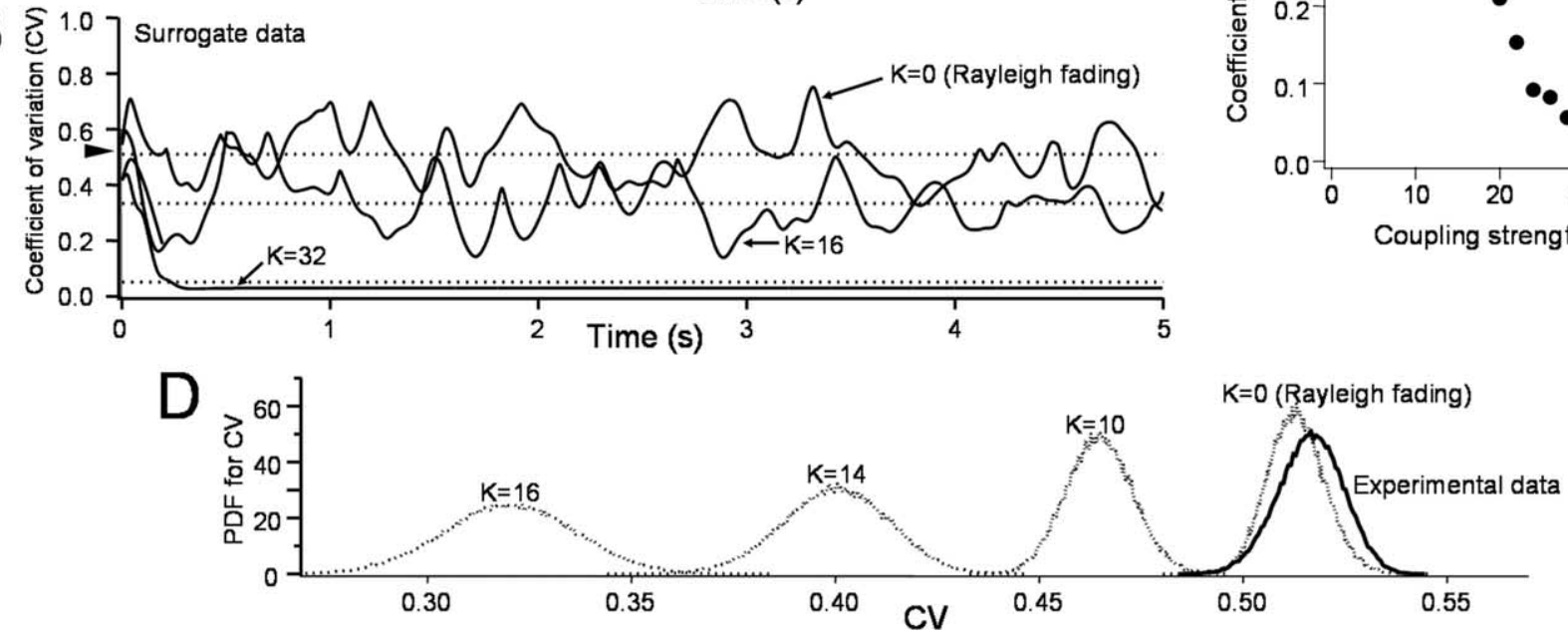

Figure 4. CV as a diagnostic tool for synchrony in neuronal signals. The variability in their modulation of a family of eight PWs obtained under the same experimental conditions (four of these traces are shown in Fig. $1 A$ ) was assessed by calculating their envelopes and, for each time $t$, the average, $\mathrm{SD}$, and $\mathrm{CV}(t) . \boldsymbol{A}$, Plot of $\mathrm{CV}(t) .\langle\mathrm{CV}(t)\rangle=0.503$ (dotted line). 0 ur total data consist of 13 such measurements equivalent to $104(13 \times 8)$ traces $(\boldsymbol{B}), \mathrm{CV}(t)$ for Kuramoto-controlled signals. The sum of 25 oscillators $(30 \pm 1.5 \mathrm{~Hz})$ following the Kuramoto model was calculated under three different coupling strengths $(K=0,16$, and 32). For each $K$, the $C V(t)$ was evaluated as in $A$ and plotted (dotted lines, $\langle C V(t)\rangle ;$ arrowheads, 0.523$)$. C, CV versus $K$ in Kuramoto systems. Surrogate data representing 13 instances of eight signals generated as the sum of 25 Kuramoto oscillators was generated for $K=0,2,4, \ldots, 42$. Resampling was used to calculate the expected $C V$ for each $K$ value. $D$, PDFs for CV obtained by resampling. Surrogate PDFs (obtained from $C$; dots) were compared with the PDF from real data (also obtained by resampling). The experimental data mean differs ( $p<$ 0.01 ) from CV simulated with $K=10$ but not from CV simulated with $K=0$.

behavior, because $K$ above a critical value $(K c)$ produces synchronization, but $K<K c$ produces only a very weak tendency to synchronization. Thus, in our numerical simulations, a null coupling strength $(K=0)$ produced Rayleigh fading, and the corresponding $\mathrm{CV}$ varied at $\sim 0.523$, as expected. When the oscillators were forced to synchronize $(K>K c)$, the $\mathrm{CV}$ of the resultant waves was of much lower value, even for $K=16$, which corresponds to a medium coupling strength that resulted in a partial synchronization $(K \approx K c)$. Initially, just after the stimulus onset, $\mathrm{CV}(t)$ is close to 0.523 , because the oscillators start with random phases; however, as synchronization takes control, the $\mathrm{CV}(t)$ drops to lower values, because most oscillators adopt similar phases (Fig. 3). After systematically exploring Kuramoto coupling strengths $(K)$ and the expected CVs, we concluded $K$ decreases as CV increases (Fig. 4C). Our set of $13 \mathrm{CV}$ measurements, obtained from empirical PWs, was compared against surrogate data obtained from Kuramoto systems using resampling techniques (Davidson and Hinkley, 1999). The PDF from the experimental data differs $(p<0.01)$ from the PDF of $K=10$ (a coupling strength that barely departs from an asynchronous state) and cannot be separated from the PDF of $K=0$ (Fig. 4D). Thus, considering our model, the value of the experimentally derived CV of PWs indicates lack of coupling between ONRs.

To further challenge our claim that CV could be considered a good indicator for random phase oscillators, we also analyzed the Matthews-Mirollo-Strogatz model (Matthews et al., 1991). The MMS model has important theoretical interest, because it considers not only phase relationships between oscillators but also allows oscillators to undergo amplitude variations. As a consequence, the MMS model exhibits a complex variety of behaviors (incoherence, locking, amplitude death, and several types of unsteady states). Thus, the MMS model has been used to check analytical methods in neuroscience (Rudrauf et al., 2006). As in the Kuramoto model, synchronization for the MMS model depends on a coupling parameter $K$ and bandwidth $(\Delta)$. Figure $5 \mathrm{~A}$ compares the variations of $\mathrm{CV}$ against $K$ for Kuramoto and MMS models at a given bandwidth $(\Delta=0.8)$. The CV for unsynchronized states lies close to 0.523 , whereas large $C V$ values $(0.65<$ $\mathrm{CV}<0.9)$ are produced by unsteady states characterized by periodic pulsating envelopes (Fig. $5 C$, trace c) very unlike PWs. As soon as the system synchronizes, however, the envelope amplitude becomes constant (Fig. 5C, trace b), and the CV drops dramatically. To globally check the behavior of $\mathrm{CV}$ with respect to synchronization, we systematically explored the $(K, \Delta)$ phase space of MMS parameters for 25 oscillators. This computation revealed that $\mathrm{CV}$ by itself reproduces the qualitative phase diagram introduced by MMS [Matthews et al. (1991), their Fig. 17] as every behavior of MMS oscillators could be identified by particular $\mathrm{CV}$ values (Fig. $5 \mathrm{~B}, \mathrm{C}$ ). In the asynchronous state, $\mathrm{CV}$ stays near 0.523 even in the presence of large changes in the amplitude of oscillators.

Rayleigh fading is an extremely robust phenomenon that does not require equal or invariant amplitudes or special temporal profiles for the individual oscillators (as the sine functions of Fig. 2 would seem to imply). Figure 6 shows examples of Rayleigh fading obtained with profiles produced by common periodic 

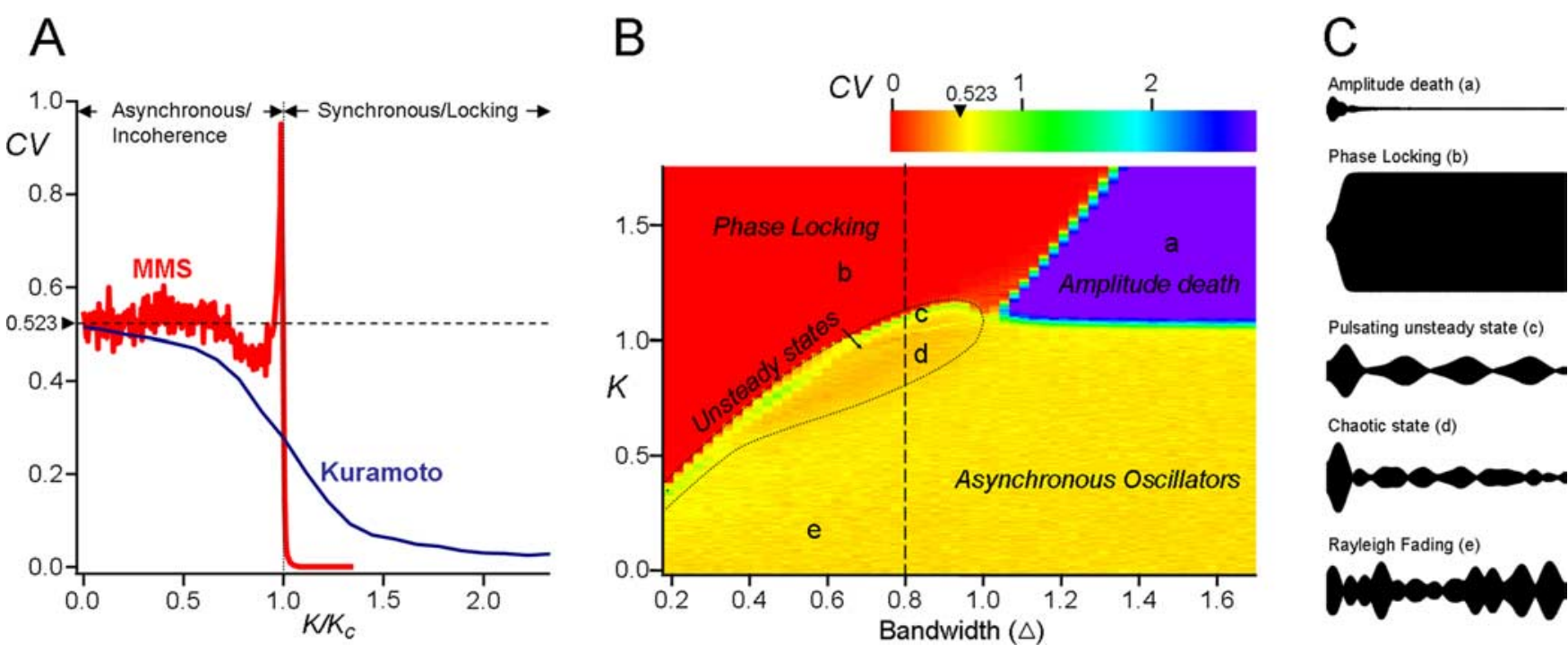

Figure 5. CV as an indicator of asynchronous oscillators in the MMS model. $A$, The CV for a population of $N=25$ oscillators were calculated for Kuramoto and MMS models and plotted against the normalized coupling strength ( $K c$, onset of synchronization for each model) at a bandwidth of $\Delta=0.8$. In both models, in the synchronized state, CVs are well below CV $=0.523$ and approach $\mathrm{CV}=0$. The maximum exhibited by $\mathrm{CV}$ in the MMS model just before $K c(C V \approx 0.9)$ is produced by pulsating oscillatory unsteady states before the onset of synchronization. $B$, $C$ olor map of $C V$ with respect to the phase space $(K, \Delta)$. The MMS phase space $(K, \Delta)$ was systematically explored $(K=0-1.8$, steps, $0.005 ; \Delta=0.2-1.7$, steps, 0.025$)$, and the $(V$ for every $(K, \Delta)$ point was color coded. The diagram shows that every behavior of the MMS regime [see Matthews et al. (1991), their Fig. 17 for a definition of phase locking, unsteady states, amplitude death, incoherence] could be identified by its $C V$ value. The line at $\Delta=0.8$ shows the transect (red trace) used in $A$. C, Representative waveforms. These five waveforms (labeled a,b,..e) show envelopes obtained at corresponding points of the $(K, \Delta)$ space.

neuronal activity. In Figure $6 A$, it was assumed that the individual oscillators were subthreshold membrane oscillations of ORNs with a temporal profile similar to an AM chirp. Alternatively, Figure $6 B$ explores the scheme that the individual oscillators responsible for $\mathrm{PW}$ generation are the spike trains produced by the ORNs (Dorries and Kauer, 2000). In both cases, waves resembling PWs were obtained. The spike trains of excited ORNs are highly regular (Fig. 6B, bottom trace) and have frequency properties that match those of PWs (Baylin, 1979; Trotier and MacLeod, 1983; Dorries and Kauer, 2000). Thus, the elementary oscillators involved in PW genesis could be neuronal subthreshold oscillations, spike trains, or a combination of both (Desmaisons et al., 1999). CV is a noise-resistant indicator, as addition of a substantive zero-mean noise processes $(\mathrm{SD}=0.05 \mathrm{rad} / \mathrm{s})$ still shows that locked states have small CVs (data not shown). Thus, although LFP data always contain noise components because the $\mathrm{CV}$ of the envelope reflects an intrinsic statistical property of the signal, the collection and averaging of enough instances of envelopes should allow the detection of the underlying CV. In contrast, our determination of the empirical CV assumes that no systematic drift exists between trials. Indirect evidence shows that this is the case, and thus the data are stationary (see below, Discussion).

\section{Discussion}

Because of the signal properties of the obtained PWs (i.e., random shape, uncorrelation with stimulus characteristics, CV close to 0.523 ) along with consideration of a simple generative model based on uncoupled oscillators, we suggest that PWs are the result of a superposition of asynchronous neuronal oscillators. Our interpretation might reconcile known anatomical data with theory. In effect, a puzzling fact in olfactory research was the conflict created by the existence of PWs and a lack of evidence for a coupling mechanism in the OE (Dorries and Kauer, 2000). Our analysis suggests an alternative path to resolve the contradiction, which arises from the implicit, but incorrect, expectation that neural oscillations must necessarily imply synchrony.

Because the observed CV is calculated by evaluating the SD across trials collected at different moments, it could be argued that this result is attributable, not to the interference produced by random oscillators, but to systematic drifts in the neural response (nonstationary effects). Because the existence of adaptation effects in the OE are well known, we followed established methods (Nikonov et al., 2002) to avoid them. In fact, the electroolfactograms (EOGs) collected following this procedure were identical, which suggests that the PWs that we collected do not have significant nonstationary effects.

Using simulated small populations of oscillators synchronized by Kuramoto or MMS dynamics, we indicated a relationship between a signal CV and the average degree of coupling among oscillators contributing to that signal. In these two models, the $\mathrm{CV}$ differentiates behaviors among oscillators (from incoherent to synchronization) and thus could be used as an indirect measurement of the underlying coupling parameter $K$. Although most modern synchronization models (like MMS) are derived from the Kuramoto model, it is still an open question whether a CV close to 0.523 could be produced by some type of synchronization regime. However, independently of the synchronization model considered, because synchronization implies necessarily a locking in the allowed values for phases and thus a restriction of the overall system variability, a low value of CV must necessarily ensue. In addition, because the amplitude variations of the oscillator are also a possible source of system variability, exploring the MMS model (where amplitudes change dynamically) provided us more general support for our claim that $\mathrm{CV}=0.523$ together with the characteristic patterns of Rayleigh fading are diagnostic features of random phase oscillators. Finally, the theoretical problem of whether a synchronization regime with a $\mathrm{CV}=0.523$ could exist or not is an interesting problem that, in conjunction 
A

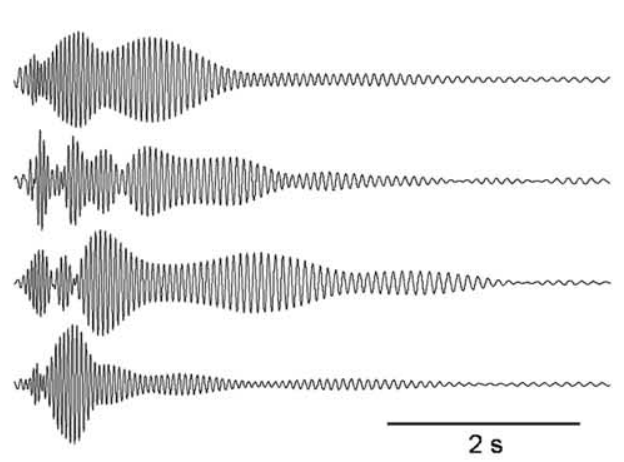

$\mathrm{B}$

B jitter

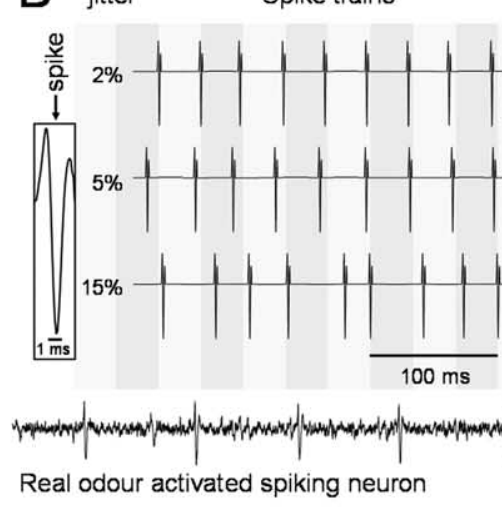

Addition of 100 spike trains (BP: $2-50 \mathrm{~Hz}$ )

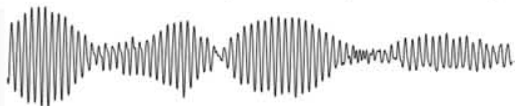

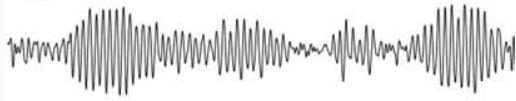

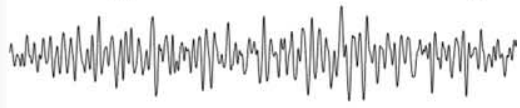

$1 \mathrm{~s}$

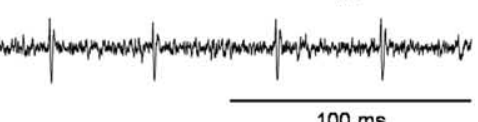

Figure 6. Simulated PWs produced by real neuronal oscillators. $A$, Examples of artificial PWs generated by the addition of 25 uncoupled oscillators. The top trace depicts the temporal amplitude profile of each elementary oscillator (10-fold magnified with respect to the other 4 traces) with an instantaneous frequency as in Figure $1 B$. These four artificial PWs resemble the amplitude and frequency profiles of real PWs (Fig. 1A). B, Artificial PWs generated as the addition of uncorrelated spike trains. One hundred artificial spike trains (left traces) were added and bandpass filtered between 2 and $50 \mathrm{~Hz}$ to obtain artificial PW signals (right traces). To obtain signals similar to PWs, the jitter level must be low ( 2 and $5 \%$ of ISI traces). High jitter levels (>10\% of ISI) destroy the amplitude modulation characteristics of PWs. Bottom trace, Portion of a typical spike train from an odorant excited in a catfish ORN with an average $\mathrm{ISI}$ of $45 \mathrm{~ms}$ and an average jitter of $1.2 \mathrm{~ms}$ ( $2.7 \%$ of ISI). The simulations presented here have a $\mathrm{CV}$ close to 0.523 , as calculated in Figure 3.

with the possible influence of nonstationary effects, deserves additional study.

Rayleigh fading, in theory, can be observed with the population of an oscillator of any size. But, because electrodes are equipotential surfaces recording the spatial average of the electric fields surrounding their conductive surface (Nunez, 1981), the rms amplitude of the signal recorded by an electrode immersed in a population of $N$ oscillators with equal amplitude $\left(V_{0}\right)$ can vary between $V_{0}$ (all oscillators in phase) and $V_{0} / \sqrt{N}$ (random oscillators). The value of the denominator $\sqrt{N}$ determines the neuronal population size where Rayleigh fading fluctuations are detectable (i.e., above the electronic recording noise level, $\sim 30 \mu \mathrm{V}$ ). Thus, Rayleigh fading could be detected in LFP recordings where the number of contributing neurons is small ( $\sim 100$ or below) but will not be observed by macroelectrodes recording from larger populations. In the OE, microelectrodes are especially suited to detect Rayleigh fading. In effect, because the OE has a single layer of ORNs (cell body diameter, $\approx 5 \mu \mathrm{m}$ ) (Erickson and Caprio, 1984) and assuming that the model of volume conduction of action potentials in a homogeneous medium can be applied (Drake et al., 1988), we concluded that microelectrodes used to record PWs detect above the noise signals from a $40-\mu \mathrm{m}$ diameter disc corresponding to $\sim 150$ ORNs, a number consistent with estimations made in other systems such as the hippocampus (Buzsaki, 2004). Furthermore, in the OE, because ORNs express only one odor receptor type, only a subset of ORNs located inside the recording space would be activated by an odor mixture. Although the exact number of neurons involved in PW generation is speculative, and a matter of current discussion, the simple division between macroscopic and mesoscopic field recordings determines the relevance and possible appearance of Rayleigh fading.

In addition to demanding that the number of oscillators be mesoscopic, Rayleigh fading imposes another requirement concerning the dispersion of frequencies of the oscillators (i.e., the oscillators must have, in every instant, similar frequency). Although it was assumed that the inherent frequency of neural oscillators is an emergent property of the neural network (Manor et al., 2000), experiments in connexin-36 knock-out mice show that uncoupled neurons from the inferior olive retain their wildtype frequencies (Long et al., 2002). Therefore, the firing fre- quency of neurons is not likely a property derived from the network but is rather an intrinsic property of their biophysics (Hutcheon and Yarom, 2000). The present model describing the generation of PWs as the addition of uncoupled oscillators may have wide implications for our understanding of neuronal population dynamics in other systems where narrow-band LFP signals have not been thoroughly analyzed. In fact, it is not that the narrow band per se that is the important condition but rather the population dispersion of frequencies should be small. Thus, because it is manifested in PWs, the frequency can change in a 1:3 ratio (Fig. $1 B$ ) (a rather extended relative bandwidth), but in each moment, the population dispersion is small.

In the model presented, the envelope of a PW carries no biologically relevant information, because it reflects the timevarying phase relationship between asynchronous oscillators and does not reflect an intrinsic property of the stimulus. Although profiles of PWs appear to be stimulus independent, the time course of the frequency change varies with the nature of the odorant and its concentration (data not shown). Thus, neural coding of odorant quality in the olfactory system could not depend on the relative phase between ORNs (because they appear to be random with respect to each other) but must exploit either the stereotyped change in frequency in ORNs or use distributed processing achieved by comparing the activity of idiotypic ORNs scattered across the $\mathrm{OE}$ that project to the same glomerulus (Mombaerts, 2006). Our results suggest that mitral cells act not as coincidence detectors but as resonant units (Hutcheon and Yarom, 2000; Izhikevich, 2001), requiring regular but not synchronous presynaptic input from ORNs.

Asynchronous neural oscillators with similar frequencies is possibly a widespread situation in the nervous system such that Rayleigh fading could be a pervasive phenomenon in many neural structures and, perhaps like the case described here for PWs, instances of Rayleigh fading could be confused with synchronization events. The analytical tool introduced here, which focuses on the envelopes of the signal and is analogous to standard procedures used in the wireless communications industry for the characterization of radio channels, could serve to evaluate the degree of synchrony in a population of neural oscillations. Finally, our model suggests that in the case of the OE, there may be a simpler interpretation of the observed data represented by PWs 
than the requirement of neural synchronization. Although we cannot rule out the possibility that ORNs are instead coupled and follow a synchronization model that because a particular covariation of phases and amplitudes produces $\mathrm{PWs}$ with a $\mathrm{CV} \approx 0.523$, our model based on uncoupled ORNs explains important experimental facts and provides an intriguing alternative hypothesis.

\section{References}

Adrian ED (1955) Potential oscillations in the olfactory organ. J Physiol (Lond) 128:21-22.

Averbeck BB, Lee D (2004) Coding and transmission of information by neural ensembles. Trends Neurosci 27:225-230.

Baylin F (1979) Temporal patterns and selectivity in the unitary responses of olfactory receptors in the tiger salamander to odor stimulation. J Gen Physiol 74:17-36.

Buzsaki G (2004) Large-scale recording of neuronal ensembles. Nat Neurosci 7:446-451.

Buzsaki G, Draguhn A (2004) Neuronal oscillations in cortical networks. Science 304:1926-1929.

Davidson AC, Hinkley DV (1999) Bootstrap methods and their applications. Cambridge: Cambridge UP.

Desmaisons D, Vincent JD, Lledo PM (1999) Control of action potential timing by intrinsic subthreshold oscillations in olfactory bulb output neurons. J Neurosci 19:10727-10737.

Dorries KM, Kauer JS (2000) Relationships between odor-elicited oscillations in the salamander olfactory epithelium and olfactory bulb. J Neurophysiol 83:754-765.

Drake KL, Wise KD, Farraye J, Anderson DJ, BeMent SL (1988) Performance of planar multisite microprobes in recording extracellular singleunit intracortical activity. IEEE Trans Biomed Eng 35:719-732.

Engel A, Fries P, Singer W (2001) Dynamic predictions: oscillations and synchrony in top-down processing. Nat Rev Neurosci 2:704-716.

Erickson JR, Caprio J (1984) The spatial distribution of ciliated and microvillous olfactory receptor neurons in the channel catfish is not matched by a differential specificity to amino acid and bile salt stimuli. Chem Sens 9:127-141.

Ermentrout GB, Chow CC (2002) Modeling neural oscillations. Physiol Behav 77:629-633.

Goodman JW (1985) Statistical optics. New York: Wiley-Interscience.

Hutcheon B, Yarom Y (2000) Resonance, oscillation and the intrinsic frequency preferences of neurons. Trends Neurosci 23:216-222.

Izhikevich EM (2001) Resonate-and-fire neurons. Neural Netw 14:883-894.

Izhikevich EM, Kuramoto Y (2006) Weakly coupled oscillators. In: Encyclopedia of mathematical physics, Vol 5, Ed 1 (Francoise J-P, Naber GL, Tsun TS, eds), p 448. Boston: Elsevier.

Jones EG (2002) Thalamic circuitry and thalamocortical synchrony. Philos Trans R Soc Lond B Biol Sci 357:1659-1673.

Lledo PM, Gheusi G, Vincent JD (2005) Information processing in the mammalian olfactory system. Physiol Rev 85:281-317.

Long MA, Deans MR, Paul DL, Connors BW (2002) Rhythmicity without synchrony in the electrically uncoupled inferior olive. J Neurosci 22:10898-10905.
Manor Y, Yarom Y, Chorev E, Devor A (2000) To beat or not to beat: a decision taken at the network level. J Physiol (Paris) 94:375-390.

Matthews PC, Mirollo RE, Strogatz SH (1991) Dynamics of a large system of coupled nonlinear oscillators. Physica D 52:293-331.

Mombaerts P (2006) Axonal wiring in the mouse olfactory system. Annu Rev Cell Dev Biol 22:713-737.

Nakazawa H, Kaji S, Ishii S (2000) Oscillatory electric potential on the olfactory epithelium observed during the breeding migration period in the Japanese toad, Bufo japonicus. Zool Sci 17:293-300.

Nikonov AA, Parker JM, Caprio J (2002) Odorant-induced olfactory receptor neural oscillations and their modulation of olfactory bulbar responses in the channel catfish. J Neurosci 22:2352-2362.

Nunez P (1981) Electric fields of the brain: the neurophysics of EEG. Oxford: Oxford UP.

Ottoson D (1956) Analysis of the electrical activity of the olfactory epithelium. Acta Physiol Scand 35:1-83.

Papoulis A (1984) Random variables and stochastic processes. New York: McGraw-Hill

Parker JM, Chang QH, Caprio J (2000) Citrate enhances olfactory receptor responses and triggers oscillatory receptor activity in the channel catfish. J Neurophysiol 83:2676-2681.

Rudrauf D, Douiri A, Kovach C, Lachaux J, Cosmelli D, Chavez M, Adam C, Renault B, Martinerie J, Le-Van-Quyen M (2006) Frequency flows and time-frequency dynamics of multivariate phase synchronization in brain signals. NeuroImage 31:209-227.

Sklar B (1997) Rayleigh fading channels in mobile digital communication systems. Part I: characterization. IEEE Comm Mag 35:90-100.

Strogatz SH (2000) From Kuramoto to Crawford: exploring the onset of synchronization in populations of coupled oscillators. Physica D 143:1-20.

Sutterlin AM, Sutterlin N (1971) Electrical responses of the olfactory epithelium of Atlantic salmon (Salmo salar). J Fish Res Board Can 28:565-572.

Suzuki N, Takahata M, Shoji T, Suzuki Y (2004) Characterization of electroolfactogram oscillations and their computational reconstruction. Chem Sens 29:411-424.

Takagi SF, Shibuya T (1961) Studies on the potential oscillation appearing in the olfactory epithelium of the toad. J Physiol (Japan) 11:23-37.

Trotier D, MacLeod P (1983) Intracellular recordings from salamander olfactory receptor cells. Brain Res 268:225-237.

Tucker D (1975a) The role of respiratory ventilation in reliably obtaining electrical waves from olfactory mucosa and nerve in response to odorous stimulation. In: 9th Japanese symposium on taste and smell (Ichioka M, ed), pp 16-17. Osaka, Japan: The Japanese Association for the Study on Taste and Smell.

Tucker D (1975b) Waves elicited from peripheral neural tissue (olfactory) in response to odorous stimulation. Biophys J 15:271a.

Varela F, Lachaux J, Rodriguez E, Martinerie J (2001) The brainweb: phase synchronization and large-scale integration. Nat Rev Neurosci 2:229-239.

White JA, Chow CC, Ritt J, Soto-Trevino C, Kopell N (1998) Synchronization and oscillatory dynamics in heterogeneous, mutually inhibited neurons. J Comp Neurosci 5:5-16. 